Vol. 6, Num. 1, 2021

\title{
USING IMPROVED HOMOPHONIC ENCRYPTION ON THE EXAMPLE OF CODE ZODIAC Z408 TO TRANSFER ENCRYPTED INFORMATION BASED ON DATA TRAFFIC
}

\author{
Oleksandr Mamro, Andrii Lagun \\ Lviv Polytechnic National University, 12, Bandera Str, Lviv, 79013, Ukraine \\ Authors'e-mail: oleksandr.mamro.kn.2017@lpnu.ua,andrii.e.lahun@lpnu.ua
}

Submitted on 03.03.2021

(C) Mamro O., Lagun A, 2021

\begin{abstract}
This article examines the improvement of homophonic encryption using the well-known Z408 cipher as an example, and analyzes their problems to further correct the main shortcomings of this typea of encryption. In the practical part, an encryption system similar to $\mathrm{Z408}$ was created and the complexity of the language analysis for this cipher was increased. The correlation dat of the number of alternative characters was used to improve the transmission of encrypted data depending on the load of the device. The more symbols and the higher the load, the fewer the number of alternative symbols will be used.
\end{abstract}

Index Terms: Zodiac, Z408, encryption, homophonic encryption, quenching, cyber-physical systems.

\section{INTRODUCTION}

Advances in modern cryptography can reliably protect information from unauthorized access and distortion of information; allow you to resolve the issue of document authorship and many other useful data protection operations. However, not everything is so cloudless. Despite the fact that energy-efficient cryptographic protection microchips are built into many IoT devices, they cannot protect against hacking or data leakage if the user of the smart device does not use them for their intended purpose or the attacker is especially persistent. For example, private keys can be read from the memory of an IoT device, calculated from a dynamic change in the supply current, or even from electromagnetic radiation.

Security of smart devices can be enhanced by installing additional security electronics - miniature components that connect peripherals to host microcontrollers or microprocessors and are responsible for personalized certificates, secure placement of private keys, and management of cryptographic elements. Or the need to improve the reliability of the confidentiality of transmitted data not at the hardware level, but at the software level. This article explores homophonic encryption systems to improve data resiliency and discusses the weaknesses of homophonic cryptography, and with the help of gamma and entropy slice increase the reliability of the cipher and improve resistance to speech analysis.

\section{OBJECT, PURPOSE AND TASKS OF THE RESEARCH}

One of the most difficult issues in the operation of homophonic encryption is the degree of protection of homophonic encryption from speech analysis, or rather the quality of masking the frequencies of occurrence of certain letters in the text. therefore, the possibility of reducing the repetition rates of text characters, and the corresponding complication of protection against cryptocurrencies of frequency analysis should be considered.

Even though it was originally a crib which helped to crack it, Z408 has other weaknesses, most notably the way it sequentially cycles through homophones ("multiple ciphertext shapes for the same plaintext character"). For example, plaintext ' $t$ ' maps to the four ciphertext homophones HI5L, and appears in the text as the sequence HI5LHI5ILHI5LHI5LHI5LHI5LI5LHL5IIHI. If you count each successful letter-to-letter transition matching the modulo-4 sequence [HI5L] as a 0.25 success event $(=26)$ and each non-match $(=8)$ as a 0.75 failure event, I believe you get a raw probability of less than 1 in a billion (i.e. of at least 26 successes from 34 events).

Frequency attack on simple substitution ciphers is a kind of greedy algorithm method. However, a frequency attack does not require a letter combination exceeding the 3rd graph ("trigraph".) The trigraph evaluation table consists of all combinations of any 3 consecutive English letters. For example, in English the combination "aba" has a frequency of $0.0075 \%$, and the combination "epa" has frequency $0.1086 \%$. To solve the problem of homophonic substitution, the cryptanalyst much higher tables of N-plots should be used which increase the probability to distinguish meaningful text from meaningless text.

This cipher rate approach assumes that the frequencies of all cipher symbols are equal balanced. Hence, the matches are selected based on "quantity" (i.e., numbers cipher characters.) This frequency-based encryption approach does not work on modified homophonic substitution cipher because the cipher character frequencies vary. In the case of the Z340 Zodiac, the frequency of all 62 encrypted characters is not consistent. Some of the 
encrypted characters have much higher frequencies than others.

Another possibility would be the columnar double transposition method. In transpositional encryption involves reordering the cipher characters. in

In the process of decryption, the reverse operation is performed. In a double column transposition, the original message is written in matrix form, which is a series of lines with a fixed length (that is, a fixed number of columns). Row and Column Order modified based on predefined keys

The aim of this work is to try to improve and analyze the influence of the shift of block structures in homophonic ciphers, their influence on the frequency analysis of the text. Additionally, there is cut off the noise in the source text before the encryption phase, which changes the language structure and its impact on language analysis.

\section{THE MAIN PART}

With homophonic substitution, the frequency of each letter is balanced to prevent any statistical-based approach.

The method uses the advantages of the existing English dictionary. Typically, the dictionary used for cryptanalysis contains approximately, but not limited to, from 100,000 to 120,000 words. This approach is to use trial and error methods on some consecutive letters of the ciphertext (ie from the letter $i$ to the letter $(i+j)$, CJ .. ( $(J+$ $J)$. The attack method looks for similar words in a dictionary that has a similar pattern to $\mathrm{Ci} . .(\mathrm{j}+\mathrm{j})$.

This method of attack assumes that the cipher is monoalphabetical. If the cipher is a polyalphabetic substitution, this attack method cannot reuse any of its successful mappings in the attack process because the polyalphabetic substitution uses a many-to-many technique. The many-tomany display technique allows a cipher character to represent many letters of plaintext, while plaintext letters can also be represented by many ciphers characters.

Another disadvantage of a dictionary-based attack is misspelled English words. The attack itself relies heavily on the existing vocabulary. Therefore, if the cipher contains non-standard or intentionally misspelled words, the attack not provides reasonable and readable relevant plaintext which can be used to increase protection against cryptocurrencies.

A homophonic cipher that uses a cipher alphabet with $\mathrm{N}$ different cipher characters has a theoretical key space of $26 \mathrm{~N}$ compared to a theoretical key space of simple replacement being only $26 ! \sim 4,033 \times 1026$. Due to the unmanageable size of this key space, a comprehensive key search is not possible.

For example, for a relatively short message that uses an alphabet of ciphers with $\mathrm{N}=50$ different cipher characters and a comprehensive search for keys using a personal computer (which can check approximately 106 keys / second) we have to take 2650 keys/106 keys/second $=5,6 * 1064$ seconds $=1.8 * 1057$ years.

However, if the message is encrypted with a simple replacement, a comprehensive key search can only be performed in $26 ! \mathrm{keys} / 106 \mathrm{keys} / \mathrm{second}=4.03 * 1020$ seconds $=1.28 * 1013$ years. The difference between the homophonic substitution key space and the simple substitution key space increases exponentially with the number of cipher characters in the cipher alphabet.

Of these strengths, we have many options to make them even better, and provide a high level of information protection by adding block offset after homophonic encryption and cutting off entropic noise from the source text.

The EMF quality factor, as a measure of the difference between the arbitrary probability density $\mathrm{p}(\mathrm{x})$ and the probability density of the normal (Gaussian) distribution $\mathrm{w}(\mathrm{x})$, is a functional that depends on the difference between the functions $\mathrm{p}(\mathrm{x})$ and $\mathrm{w}(\mathrm{x})$, ie

$\mathrm{Y}=\mathrm{Q}\left\{\mathrm{p}(\mathrm{x})-\mathrm{w}\left(\mathrm{x}, \mathrm{a}_{1}, \mathrm{a}_{2}, \ldots, \mathrm{a}_{\mathrm{n}}\right\}\right.$,

where $a_{1}, a_{2}, \ldots, a_{n}$ - parameters that characterize the distribution density $\mathrm{w}(\mathrm{x})$. This definition involves calculating the functional by minimizing it by the parameters $a_{1}, a_{2}, \ldots, a_{n}$ and to obtain values of the functional close to zero. Therefore, to find and select weak links and their subsequent removal, we use

$$
H=-\sum_{i} P_{i} * \log _{2} P_{i}
$$

where $P_{i}$ is the probability of the $i$-th result.

For block encryption we can take the mode of tempering. This mode has no disadvantages of the simple replacement mode. Gamma mode is so called because it uses gamma - a pseudo-random sequence, which in each round is composed of module 2 with plaintext.

The gamma is formed with a synchronization S pseudo-random sequence, which changes with each iteration and is encrypted in the mode of simple replacement, then converted into gamma and superimposed on the plaintext of Fig. 1.

To improve homophonic encryption on the example of the code Z408, we have used the practical implementation of the code Z408.

Starting with the clipping of entropic noise from the source text, as mentioned above, using the formula of the quality factor of EMF noise, we add to the source text such processing of Fig. 2.

Thus we receive the source text passed through noise and receive the text with the brought down structure of language.

There is an example of encryption process:

Source text: I like to bill people For my investigation

Received text: IlietobilpeoplFormyivstgation

Rejecting $25 \%$ of our text, we still have a readable structure for humans, and the language structure itself is no longer $100 \%$ correct, which further adds complexity to language analysis.

Plaintext: There are many variations of passages of Lorem Ipsum available but the majority have suffered alteration in some form by injected humor or randomized words which don't look even slightly believable If you are going to use a passage of Lorem Ipsum, you need to be sure 

data traffic

there isn't anything embarrassing hidden in the middle of text.

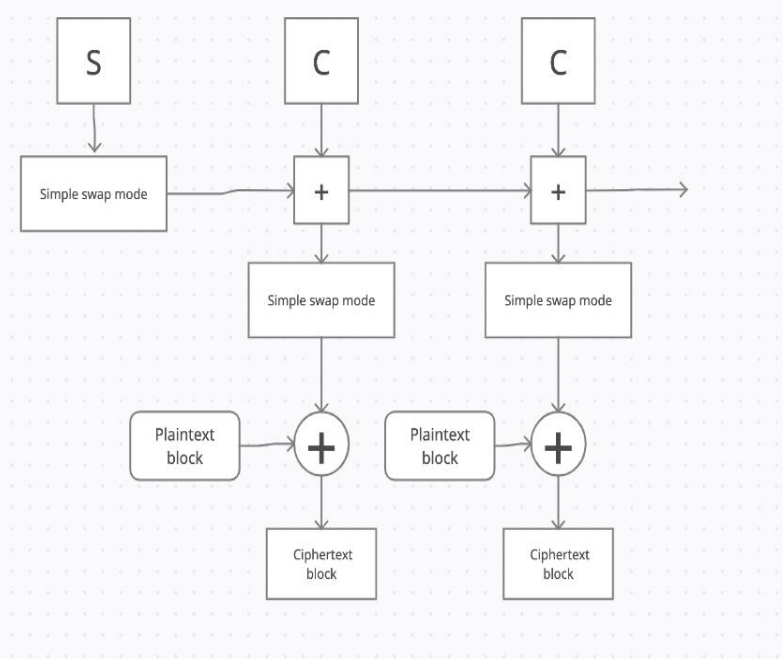

Fig. 1. The scheme of manipulation of blocks by means of hardening

All the Lorem Ipsum generators on the Internet tend to repeat predefined chunks as necessary making this the first true generator on the Internet.

It uses a dictionary of over Latin words combined with a handful of model sentence structures to generate Lorem Ipsum which looks reasonable.

The generated Lorem Ipsum is therefore always free from repetition injected humor or non-characteristic words etc.

\section{With noise text:}

ThreareanyvaraionsofpssaesoforemIpsmaailablbutthe majriyhavsuferealertioninomeormbyinjeteumourorandomis dworswichdotloovenslihtybeievabeIfyouareoingtouseaassag eoforemIpsm,youneetobesurheresntanyingembarasighiddni nthemiddloftxtAlltheLormpsumgneratosonthentenettedtoree atpredenedhunksasncesaryakingtisthefirttueeneraorontheInt rntItussaictonaryofoveLainworscombiewthahndfulofodelsen tnctructrestogeneaeoremIsumwhicloksreasnbleThegeneaed LremIsumistherefrawaysfrefroretitionijectdhumuroronracteristicworsetc

Encrypted text: 451856506601113742601113 4286879976050679476289342828715156202850 520113758154436685436353601406967202578 $\begin{array}{llllllllllllll}406 & 923 & 596 & 764 & 809 & 113 & 675 & 809 & 675 & 725 & 305 & 187 & 148\end{array}$ 992473113745353967644394923332154725598 58374275860907758742539967436418762418577 4735833365444066752887626866992756758775 4738937755441157116087637636940629850811 2257735357822816284946811970187809336970 99760141885090776244830519167575829758384 59667575876215619171533560 .... (abbreviated)

Frequency analysis of Fig. 3, 4 for the implemented step of changing the structure shows us that the result did not change to tangible values (we can even level by reducing repetitions referring to error), and the main purpose of this step was to minimize text coherence. This means that frequently used repetitions of English words 11, $\mathrm{nn}$, or syllables are caught by language analysis much worse.

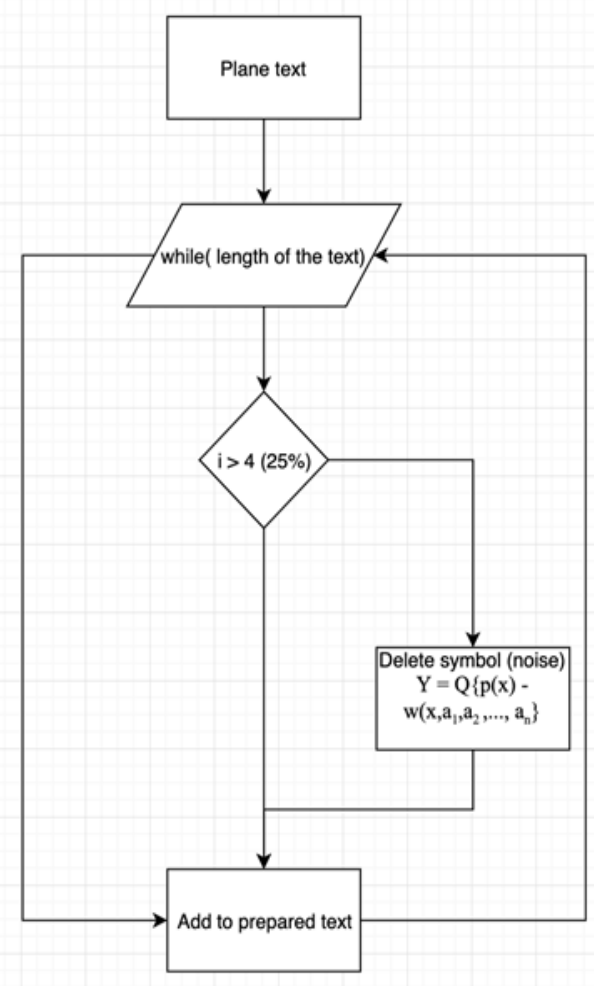

Fig. 2. Clipping of entropic noise from the source text scheme

The next step is to skip our received text, after the deformation of the language structure, through block encryption by the method of gamification, for this we need to go through several steps:

The first is to extend our alphabet to registers and special characters for further use in tempering:

Alphabetic symbols structure for now: abcdefghijklmnopqrstuvwxyzABCDEFGHIJKLMNOPQRS TUVWXYZ=/+-1234567890@; ; <> : !!?*

And it is necessary to add functionality for gamification with the corresponding XOR functionality as was mentioned in Fig. 1 scheme.

Example of implementation Code snippet has shown on fig. 5.

Thus we get the following text:

GUM text: $E 4 G T=G T 14 h J 4 c=<^{\wedge} 2 F^{\wedge}:$ EPO4VO: WPGT1 I $a O @ P=<]=7]>@ e H=T 14$ [3Ge4CTI3TNOU =9eN4e5; X2: I93^17h5; [? 0U4@^ $I G \wedge N G P 21 \wedge 1<T 3 G U O=X ?=\wedge 2 A \wedge 3>$ TJ0b52YHLS99X97] $9 \mid$ WE: $d=G T ;<; A \wedge I F T=$ EPOFP ::WP: c9EbI8h3@_91e37TOGTH0c9F_H4_H = 
$X ; 0 S=G c O<;=X 80 \_$e $\left.40 \mid 51\right]$ 9: WHMe] 9] $\mathrm{H}=\mathrm{TP}$ : c9 I aI8V9; TN4cO: $H_{2}=$ T2AT20e9; UH: c9ETHEc91X20U?@_7FPO; T9FPNL I => X; AYOAY9 $<$ cOAeIOV9; $c=A^{\wedge}$ 3E4G4; e408; ; PN: WJ0cP4e5: c8FR38S5; XH=P =; U: @] 33 130]O0_9;RN@RH@ $c 9 F e 32 T 20 c=Y \wedge N 88 L F d K<R 49 \wedge 3>c 94 b 3$; P9aY92_9GPHO $~ N O ~ \mid ~ L F d 1<b 40 c 93:$ I NOT5AX3; X26e91YI8 ^ I: c3; $\alpha ?=P N 4 R H 0 X H 6 f 3 G U 9 A R$

\section{Symbols Usage Plane Text (620)}

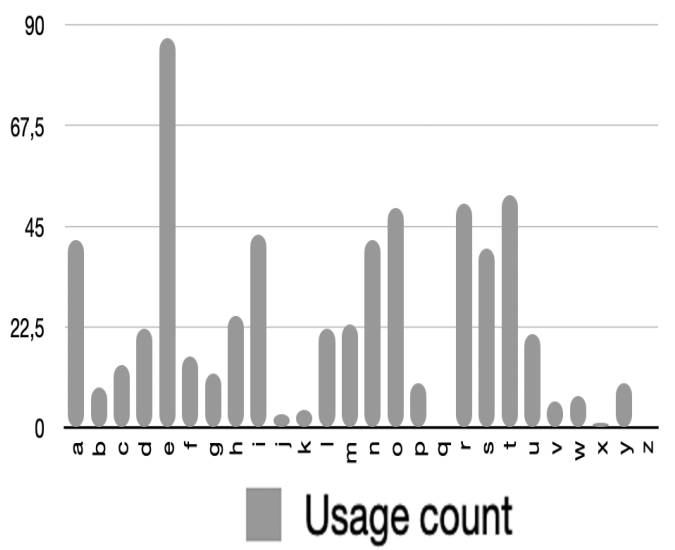

Fig. 3. Symbolic repetition of frequency analysis of the source text

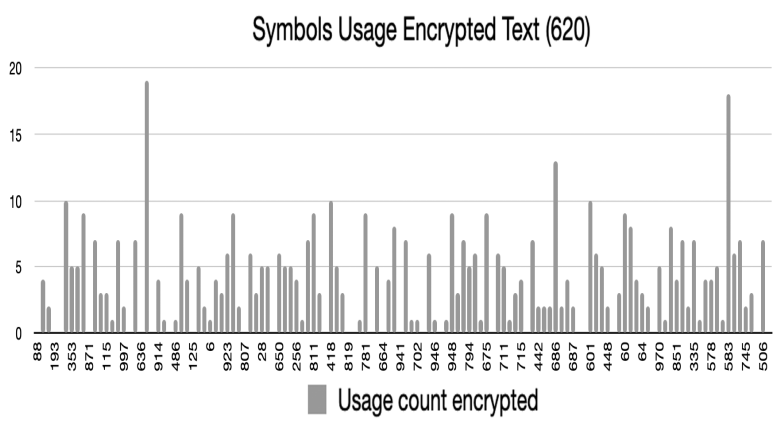

Fig. 4. Symbolic repetition of frequency analysis in ciphertext after beating the language structure of the source text

Using the received text in our homophonic encryption Z408 we receive absolutely destroyed language structure which is exposed to language analysis many times more difficult, and more exact information is resulted on fig. 6 .

Encrypted text: 023568772030654565818633 610663975243162201526360278959456103253 7333796131456918082701368763210733797952 791703306188373511494405791136759345400 42650093254665838840072011080966030630694 11034569430118088155145652589267959559206 99122231378414598345646983388518983608545 18164775958375970365876281179733306881137 299583172287448481534136713199334885647
99420843014789308816449565101470110344222

30654513673570352731297448388877951306103

19173352380818024251719174647730626334388

7821422676033830785500697863658101332874 $35199 \ldots$... (abbreviated)

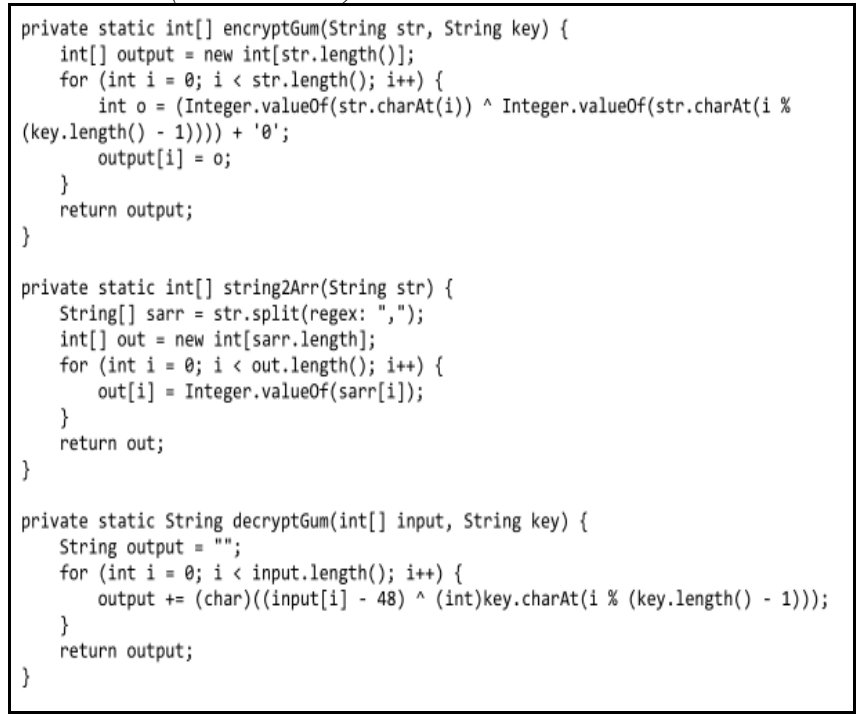

Fig. 5. Hardening functionality

\section{Symbols Usage Encrypted Text (620)}

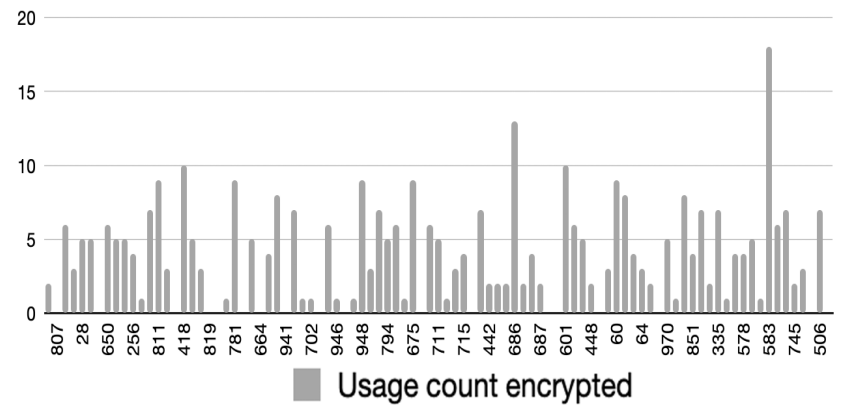

Fig. 6. Graph of language analysis after the destruction of the language structure

And finally, after ours different practical investigations, we can see that homophonic encryption with very simple additional operations can be very reliable and protected from external interference scripting. Additionally, no complex computational operations occurred, so this encryption method does not require high machine power. Analyzing the graph of symbolic repetition of frequency analysis in encrypted text (Fig. 6) we can observe a decrease in frequencies by another 23\% (compared to homophonic encryption without additional action.) But a great achievement is the lack of language structure, which makes this method of encryption analysis.

Usage in a cyber-physical system (transmitter encrypting incoming source information).

With high workload and high volumes of data, they are encrypted using fewer alternative characters and reduce 
the protection of the original text. With a low load on the transmitter, the data is encrypted using the maximum available number of alternative characters for the device, which the transmitter's RAM allows. Thus, the highest protection for the original text is preserved.

With this approach, we can reduce the load on the transmitter and encryption device (on fig. 7) based on the amount of incoming data.

With a large amount of incoming data, the level of protection of the original information will be reduced, but due to the amount of this data, it will be very difficult to find the "necessary" information package.

With a small amount of information, the required information package will be much easier to find, but due to the high level of protection of each information package, it will be much more difficult to gain access to the package (decrypted) (correlated from alternative pairs of characters).

In this case, all data passing through all involved communication channels, including the text of the message, as well as technical information about its routing, communication protocol, etc., undergoes a cryptographic transformation.

The participants in the data transfer (for example, a switcher) will decrypt the incoming stream in order to process it, then encrypt it and transmit it to the next network node, which is an effective means of protecting information in computer networks. Since all information is encrypted, a potential attacker has no additional information about who is the source of the data, to whom it is sent, and so on.

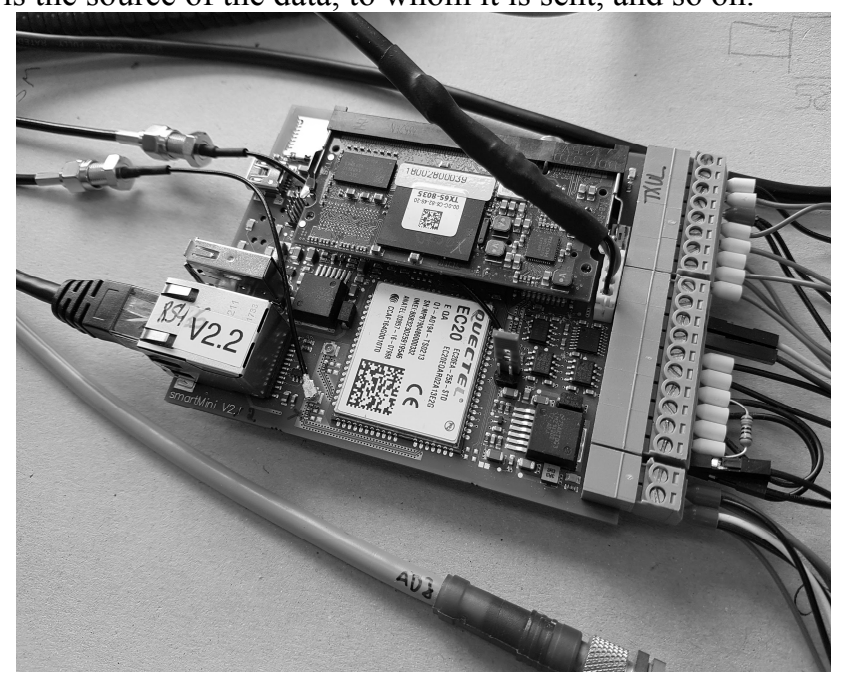

Fig. 7. Data transmitter and encoder

The simplified system (on fig. 8) does not include methods for monitoring and collecting data for process control and processing of passing data. Also, for a simplified system, you can use a less powerful processor and less memory, because all information is processed by breaking it down into sectors, which allows you to launch this device into production with lower financial costs. If there is a need for more efficient and faster processing, it could be done by raising the amount of memory used, which will expand the capacity of the encryption blocks and reduce the number of sectors required for processing.

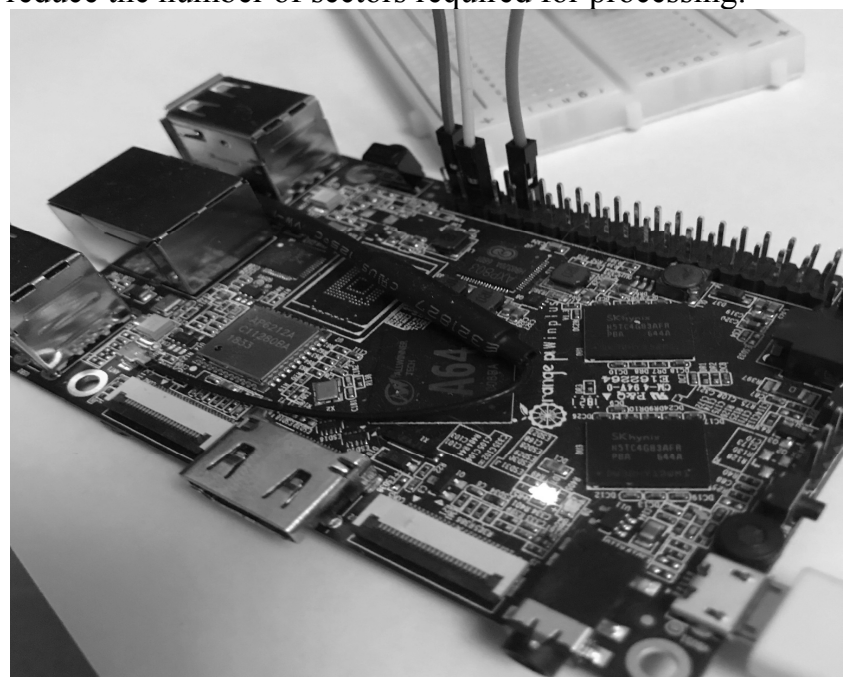

Fig. 8. Example of a simplified data transmitter and encoder system

\section{CONCLUSIONS}

Today, computer security is a house of cards that can crumble at any moment. So many weak products have not yet been hacked just because they are underutilized. Once they become widespread, they will attract criminals.

And in the future, as commerce and communications become increasingly tied to computer networks, cryptography will become vital. But the cryptographic tools on the market do not provide the level of protection promised in the advertisements. In the end, the security of these products will determine the victory in the crypto product market.

In this article, homophonic encryption was considered, and encryption was improved using entropy text slicing and block encryption. The analysis of the homophonic cipher with improvements on the example of the known cipher Z408 was realized and by its analogy the automatic algorithm of ciphering of the derived text was developed.

For example, it was proved that the entropy slice of the derived text complicates language analysis, prevents unauthorized access to information and additional block operations completely close the possibility of cryptocurrencies of frequency analysis due to the complete absence of frequencies of letters in the text (due to impaired language structure).

These studies have been used to create an improved method for transmitting encrypted data, depending on the workload of the physical transmitter.

\section{References}

[1] Polyalphabetic cipher. [Online]. Available: https://en.wikipedia.org/wiki/Polyalphabetic_cipher.

[2] Analysis of the Zodiac 340-cipher. [Online]. Available: https://scholarworks.sjsu.edu//cgi/viewcontent.cgi?referer $=\mathrm{ht}$ tps:// 
www.google.com $/ \&$ httpsredir $=1 \&$ article $=4566 \&$ context $=$ etd theses.

[3] $\bar{D}$. Welsh, "Codes and Cryptography". Clarendon Press, Oxford, 1988.

[4] Elonka Dunin, Klaus Schmeh, "Code Breaking and Cryptograms", A Practical Guide, Robinson, 2020, ISBN 978-1-472-14421-8.

[5] H. Beker and F. Piper, "Cipher Systems", Northwood Books, London, 1982.

[6] Bruce Schneier, "Applied Cryptography. Protocols, Algorithms, and Source Code in C", John Wiley \& Sons, 1996.

[7] Welsh, Dominic (1988). Codes and Cryptography, Oxford University Press, A brief textbook intended for undergraduates.

[8] Smart, Nigel (2004). Cryptography: An introduction ISBN 0-07-709987-7. Similar in intent to Applied Cryptography but less comprehensive.

[9] Patterson, Wayne (1987). Mathematical Cryptology for Computer Scientists and Mathematicians, Rowman \& Littlefield, ISBN 0-8476-7438-X

[10] Mao, Wenbo (2004). Modern Cryptography Theory and Practice ISBN 0-13-066943-1. An up-to-date book on cryptography.

[11] Gaines, Helen Fouché (1939). Cryptanalysis, Dover, ISBN 0-486-20097-3. Considered one of the classic books on the subject, and includes many sample ciphertext for practice.

[12] Katz, Jonathan and Lindell, Yehuda (2007 and 2014). Introduction to Modern Cryptography, [2] CRC Press.

[13] Falconer, John (1685). Cryptomenysis Patefacta, or Art of Secret Information Disclosed Without a Key. One of the earliest English texts on cryptography.

[14] Aumasson, Jean-Philippe (2017), Serious Cryptography: A Practical Introduction to Modern Encryption. No Starch Press, 2017.

[15] Anderson, Ross - Security Engineering, Wiley, ISBN 0-47138922-6 (online version), advanced coverage of computer security issues, including cryptography. [Online]. Available: https://www.cl.cam.ac.uk/ rja14/book.html

[16] Boak, David G. A History of U.S. Communications Security (Volumes I and II); the David G. Boak Lectures, National Security Agency (NSA), 1973 [Online] Available: https://www.governmentattic.org/18docs/Hist_US_COMSE C_Boak_NSA_1973u.pdf

[17] Budiansky, Stephen, Code Warriors: NSA's Codebreakers and the Secret Intelligence War Against the Soviet Union (Knopf, 2016). (ISBN 0385352662)

[18] Marks, Leo, Between Silk and Cyanide: a Codemaker's Story, 1941-1945, (HarperCollins, 1998). (ISBN 0-68486780-X)

[19] Levy, Steven - Crypto: How the Code Rebels Beat the Government - Saving Privacy in the Digital Age (ISBN 014-024432-8)

[20] Kozaczuk, Władysław, Enigma: How the German Machine Cipher Was Broken, and How It Was Read by the Allies in World War II, edited and translated by Christopher Kasparek, Frederick, MD, 1984

[21] Yardley, Herbert, The American Black Chamber (ISBN 0345-29867-5), a classic 1931 account of American codebreaking during and after World War I; and Chinese Black Chamber: An Adventure in Espionage (ISBN 0-395-346487)

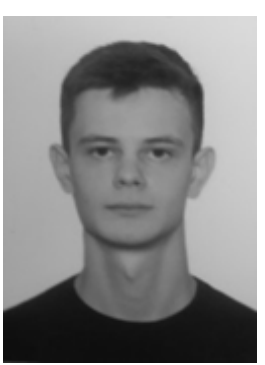

Alexander Mamro is a student of the Department of Information Systems and Technologies of the Institute of Enterprise and Advanced Technologies of Lviv Polytechnic National University. His research interests include software development for databases, business requirements analysis and software specification, design and research of cryptographic systems, building management of organizational processes and

business tasks.

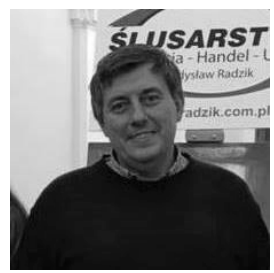

Andrii Lagun is a Head of the Department of Information Systems and Technologies of the Institute of Enterprise and Advanced Technologies of Lviv Polytechnic National University. In 2002 she obtained $\mathrm{PhD}$ in the field of "Elements and Devices of Computer and Control Systems" (degree of Candidate of Technical Sciences). His research interest includes Information theory, Investigation of Cryptographic and Steganographic algorithms and systems, Using Wavelet and Fourier Transforms in Cyberphysical and Cybersecurity Systems and also in the Internet of Things, Different Types of web and mobile Applications. 